\title{
Approximation of Wide-Sense Stationary Stochastic Processes by Shannon Sampling Series
}

\author{
Holger Boche, Senior Member, IEEE, and Ullrich J. Mönich, Student Member, IEEE
}

\begin{abstract}
In this paper, the convergence behavior of the symmetric and the nonsymmetric Shannon sampling series is analyzed for bandlimited continuous-time wide-sense stationary stochastic processes that have absolutely continuous spectral measure. It is shown that the nonsymmetric sampling series converges in the mean-square sense uniformly on compact subsets of the real axis if and only if the power spectral density of the process fulfills a certain integrability condition. Moreover, if this condition is not fulfilled, then the pointwise mean-square approximation error of the nonsymmetric sampling series and the supremum of the mean-square approximation error over the real axis of the symmetric sampling series both diverge. This shows that there is a significant difference between the convergence behavior of the symmetric and the nonsymmetric sampling series.
\end{abstract}

Index Terms-Approximation error, mean-square convergence, nonsymmetric sampling series, power spectral density, Shannon sampling series, stochastic process, uniformly bounded, weak-sense stationary.

\section{INTRODUCTION}

$\mathbf{S}$ AMPLING theory has a long history [1] and proved useful in many applications, especially in communications and information theory [2]. The conversion of discrete-time signals into continuous-time signals is a fundamental tool in information theory and has been used in many classical papers. The initial sampling theorems were only stated for the relatively small class of bandlimited signals with finite energy [2]. Since the beginnings of sampling theory, many extensions have been considered [3]: irregular sampling [4], [5], multidimensional sampling [6], and sampling theorems for larger signal spaces [7], only to mention a few.

In addition to the reconstruction of deterministic signals from their samples, the reconstruction of stochastic processes is important because they often appear in the modeling of physical processes. By now many results for the reconstruction of stochastic processes have been presented. In [8], Balakrishnan gave a rigorous proof that the Shannon sampling series converges in the mean-square sense for bandlimited wide-sense

Manuscript received April 01, 2008; revised August 05, 2010. Date of current version November 19, 2010. This work was supported by the German Research Foundation (DFG) under Grant BO 1734/9-1.

H. Boche was with Technische Universität Berlin, Heinrich Hertz Chair for Mobile Communications, D-10578 Berlin, Germany. He is now with Technische Universität München, D-80333, Munich, Germany (e-mail: boche@tum.de).

U. J. Mönich is with Technische Universität Berlin, Heinrich Hertz Chair for Mobile Communications, D-10578 Berlin, Germany (e-mail: ullrich.moenich@mk.tu-berlin.de).

Communicated by H. Bölcskei, Associate Editor for Detection and Estimation.

Digital Object Identifier 10.1109/TIT.2010.2080510 stationary stochastic processes that have either a spectral density or a spectral distribution, which is continuous at the endpoints of the spectrum. The almost sure convergence of the Shannon sampling series with oversampling was proved in [9] for wide-sense stationary processes. Zakai [7] generalized the notion of bandlimited processes and proved almost sure convergence for this new class. Later, [10] and [11] extended the results to hold for a broader classes of nonstationary second order processes. In [12], Brown analyzed the truncation error for bandlimited wide-sense stationary stochastic processes with continuous power spectral density, and in [13] upper bounds for the truncation error were derived for stochastic processes which are bandlimited in the sense of Zakai under the assumption of a guard band. The problem of reconstructing a bandlimited stochastic processes from nonequidistant samples was investigated in [14]. In [15], Habib analyzed sampling representations of bounded linear operators acting on stochastic processes that are bandlimited in the sense of Zakai [7] and Lee [16]. The approximation behavior of sampling representations for a larger class of bounded linear translation-invariant operators and deterministic signals was analyzed in [17]. For a general overview of sampling theorems for stochastic processes, see for example [3], [6, Ch. 9], and [18].

In this paper, we analyze and compare the convergence behavior of the symmetric and the nonsymmetric Shannon sampling series for the class of bandlimited wide-sense stationary stochastic processes, that have an absolutely continuous spectral measure, i.e., that have a power spectral density. We show for this class, which is smaller than the class considered in [15], that the nonsymmetric sampling series can have convergence problems. This result is interesting because in some early publications [19], [20], [12] claims were made that suggested that there is no difference in the convergence behavior of the symmetric and the nonsymmetric sampling series.

The paper is organized as follows. In Section II we introduce notation and motivate the problems which will be analyzed subsequently in Section III. In Section IV, we discuss the integrability condition on the power spectral density that was found to be crucial for the convergence behavior of the nonsymmetric sampling series. We conclude the paper with Section V.

\section{NOTATION AND MOTIVATION}

We restrict our analyses to wide-sense stationary processes, i.e., the class of continuous-time, complex valued stochastic processes $X=X(t)$ with zero mean $\mathbb{E}(X(t))=0$ and finite second moment $\mathbb{E}\left(|X(t)|^{2}\right)<\infty$ for all $t \in \mathbb{R}$, and whose correlation function $\Gamma\left(t, t^{\prime}\right)=\mathbb{E}\left(X(t) X^{*}\left(t^{\prime}\right)\right)$, where * denotes the complex conjugate, is only a function of the difference $t-t^{\prime}$. 
This enables us to define the correlation function as a function of one variable

$$
R(\tau):=\mathbb{E}\left(X(\tau) X^{*}(0)\right)
$$

and it can be easily seen that $R$ is nonnegative definite. Furthermore, we assume that $X$ is mean-square continuous, which implies that $R$ is continuous. Then the correlation function $R$ has the representation

$$
R(\tau)=\frac{1}{2 \pi} \int_{-\infty}^{\infty} \mathrm{e}^{i \omega \tau} \mathrm{d} \mu(\omega)
$$

for a positive and finite measure $\mu$. For details and further facts about wide-sense stationary processes, we refer to the standard literature, for example [21] or [22]. We additionally assume that the measure $\mu$ is absolutely continuous with respect to the Lebesgue measure $\lambda$, which implies that there exists a function $S \in L^{1}(\mathbb{R})$ such that $\mathrm{d} \mu=S \mathrm{~d} \lambda \cdot L^{p}(\mathbb{R}), 1 \leq p<\infty$, denotes the space of complex-valued measurable functions, defined on $\mathbb{R}$ that are Lebesgue integrable to the $p$ th power. Furthermore, since $\mu$ is positive, it follows that $S(\omega) \geq 0$ almost everywhere (a.e.). $S$ is called the power spectral density. We say the wide-sense stationary process $X$ is bandlimited with bandwidth $\sigma>0$, if $R$ can be extended to an entire function, and for all $\epsilon>0$ there exists a constant $C(\epsilon)$ with $|R(z)| \leq$ $C(\epsilon) \exp ((\sigma+\epsilon)|z|)$ for all $z \in \mathbb{C}$. It follows that almost all sample functions are entire functions of exponential type at most $\sigma$ [9].

Definition 1: We call a bandlimited wide-sense stationary mean-square continuous process an I-process if its correlation function $R$ has the representation

$$
R(\tau)=\frac{1}{2 \pi} \int_{-\pi}^{\pi} S(\omega) \mathrm{e}^{i \omega \tau} \mathrm{d} \omega
$$

for some $S \in L^{1}[-\pi, \pi]$. Further, if $R$ has the representation (1) then the function $S$ is unique. Note that the fact $S(\omega) \geq 0$ a.e. will be essential for the proofs. "I" stands for integrability.

In this paper we analyze the reconstruction of I-processes $X$ from their samples $\{X(k)\}_{k \in \mathbb{Z}}$, using the symmetric Shannon sampling series

$$
\sum_{k=-N}^{N} X(k) \frac{\sin (\pi(t-k))}{\pi(t-k)}
$$

$N \in \mathbb{N}$, and the nonsymmetric Shannon sampling series

$$
\sum_{k=-M}^{N} X(k) \frac{\sin (\pi(t-k))}{\pi(t-k)}
$$

$M, N \in \mathbb{N}$. One basic question, which has been studied from the beginning, is how well the I-process can be approximated in the mean-square sense by using (2) or (3), and whether the approximation error converges to zero if more and more samples are used for the approximation, i.e., if $N$, or $M$ and $N$, tend to infinity in (2) and (3), respectively. Of course, such a behavior would be desirable and is intuitively expected for the symmetric, as well as the nonsymmetric, sampling series. The early researchers who studied the convergence behavior of the
Shannon sampling series for stochastic processes were probably also led by this intuition, and therefore thought that there is no difference in the convergence behavior of the symmetric and the nonsymmetric sampling series.

As for the symmetric sampling series, it is well known [12] that, for all I-processes $X$ and $0<T<\infty$ fixed, we have

$\lim _{N \rightarrow \infty}\left(\max _{t \in[-T, T]} \mathbb{E}\left|X(t)-\sum_{k=-N}^{N} X(k) \frac{\sin (\pi(t-k))}{\pi(t-k)}\right|^{2}\right)=0$

i.e., the variance of the reconstruction error of the symmetric Shannon sampling series is bounded on all compact subsets of $\mathbb{R}$ and converges to zero for $N \rightarrow \infty$. It was believed that this result is equally true for the nonsymmetric Shannon sampling series. However, this is-as we will see-not the case.

We begin our discussion with the convergence behavior of the nonsymmetric sampling series for deterministic signals. Of course, when one considers the convergence of sampling series for deterministic signals, one must clearly specify the nature of the signals being employed in the series. Two commonly used classes of bandlimited signals are the Paley-Wiener spaces $\mathcal{P W}_{\pi}^{1}$ and $\mathcal{P} \mathcal{W}_{\pi}^{2}$. In general we denote by $\mathcal{P} \mathcal{W}_{\pi}^{p}, 1 \leq p \leq$ $\infty$ the Paley-Wiener space of signals $f$ with a representation $f(z)=1 /(2 \pi) \int_{-\pi}^{\pi} g(\omega) \mathrm{e}^{i \omega z} \mathrm{~d} \omega, z \in \mathbb{C}$, for some $g \in$ $L^{p}[-\pi, \pi]$. Consequently, $\mathcal{P} \mathcal{W}_{\pi}^{2}$ is the space of all bandlimited signals $f$ with bandwidth $\pi$, whose Fourier transform $\hat{f}$ is in $L^{2}[-\pi, \pi]$, and $\mathcal{P} \mathcal{W}_{\pi}^{1}$ the space of all bandlimited signals $f$ with bandwidth $\pi$, whose Fourier transform $\hat{f}$ (taken in the distributional sense) is in $L^{1}[-\pi, \pi]$. According to the Cauchy-Schwarz inequality we have $\mathcal{P} \mathcal{W}_{\pi}^{2} \subset \mathcal{P} \mathcal{W}_{\pi}^{1}$.

In the introduction of the paper [20], Brown argues that the nonsymmetric Shannon sampling series

$$
\left(S_{M, N} f\right)(t):=\sum_{k=-M}^{N} f(k) \frac{\sin (\pi(t-k))}{\pi(t-k)}
$$

is convergent, i.e., that the truncation error $f(t)-\left(S_{M, N} f\right)(t)$ converges to zero, for all real-valued signals $f \in \mathcal{P W}_{\pi}^{1}$ and all $t \in \mathbb{R}$ as $M$ and $N$ tend to infinity. Although we have the convergence $\lim _{M, N \rightarrow \infty}\left|f(t)-\left(S_{M, N} f\right)(t)\right|=0$ for all signals $f \in \mathcal{P} \mathcal{W}_{\pi}^{2}$ and all $t \in \mathbb{R}$, convergence cannot be guaranteed for signals in $\mathcal{P W}_{\pi}^{1}$. In [23] a real-valued signal $f_{1} \in \mathcal{P} \mathcal{W}_{\pi}^{1}$ was constructed such that

$$
\limsup _{M, N \rightarrow \infty}\left|f_{1}(t)-\left(S_{M, N} f_{1}\right)(t)\right|=\infty
$$

for all $t \in \mathbb{R} \backslash \mathbb{Z}$.

The double limit $\lim _{M, N \rightarrow \infty}$ in the equations above is defined as usual [24]. For a double sequence $\left\{a_{M N}\right\}_{M, N \in \mathbb{N}} \subset \mathbb{C}$, we write $\lim _{M, N \rightarrow \infty} a_{M N}=c$ if for all $\epsilon>0$ there exists a $n \in \mathbb{N}$ such that $\left|a_{M N}-c\right|<\epsilon$ for all $M>n$ and $N>n$. Moreover, we write $\lim \sup _{M, N \rightarrow \infty} a_{M N}=\infty$ if for all $K>0$ there exist two natural numbers $M$ and $N$ such that $a_{M N} \geq K$.

The main goal of [20] was to estimate the truncation error magnitude $\left|f(t)-\left(S_{M, N} f\right)(t)\right|$, as a function of $M, N$, and $t$. Despite the imprecise claims in the introduction, which are 
too strong in this generality, the theorems in [20] are correct because an additional guard band assumption was made in the theorems. According to the guard band assumption, only signals $f \in \mathcal{P W}_{r \pi}^{1}, 0<r<1$, are considered, which means that $\hat{f}$ must vanish outside the interval $[-r \pi, r \pi]$. This corresponds to an oversampling of the signal because the sampling rate is higher than the Nyquist rate. Under this guard band assumption, the nonsymmetric sampling series $\left(S_{M, N} f\right)(t)$ converges for all signals $f \in \mathcal{P W}_{r \pi}^{1}$, and all $t \in \mathbb{R}$, and it is possible to give convergence rates. Further papers which derive upper bounds on the truncation error of the nonsymmetric Shannon sampling series under the assumption of a guard band are [25] and [26].

All upper bounds in [20], [25], and [26] diverge as the guard band is reduced to zero, i.e., as $r$ goes to 1 . It seems likely that various authors thought that this divergence of the upper bounds does not reflect the actual convergence behavior of the nonsymmetric sampling series and that the techniques used to establish these bounds were too weak to analyze the "true" convergence behavior. However, the reason for the divergence lies clearly in the fact that there are signals in $\mathcal{P} \mathcal{W}_{\pi}^{1}$ for which the nonsymmetric Shannon sampling series diverges. Additional requirements like the guard band requirement are in fact necessary for the convergence of the nonsymmetric Shannon sampling series and not only a convenient means to obtain tighter bounds or better convergence rates. A passage in [20] substantiates the suspicion that this was not known: "Thomas et al. ... found that tighter upper bounds could be obtained by making the additional assumption that the signal spectrum $F$ vanishes in the so-called 'guard band' from $-\pi$ to $-\pi r$ and from $\pi r$ to $\pi$, where $r$ is some fixed real number satisfying $0<r<1$."

At that time, one was probably convinced that the claim in the introduction of [20] about the convergence of the nonsymmetric Shannon sampling series for signals in $\mathcal{P W}_{\pi}^{1}$ was true, because in [19] a flawed proof was given, which indicates the convergence of $\left(S_{M, N} f\right)(t)$ for all signals in $\mathcal{P} \mathcal{W}_{\pi}^{1}$. The problem in the proof arises because

$$
\sum_{k=-M}^{N} \mathrm{e}^{i \omega k} \frac{\sin (\pi(t-k))}{\pi(t-k)}
$$

cannot be bounded above on $[-\pi, \pi]$ by a function in $L^{\infty}[-\pi, \pi]$ independently of $M$ and $N$, and thus the application of Lebesgue's dominated convergence theorem, as done in [19], is not justified.

In [12], the imprecise statement about the convergence behavior of the nonsymmetric Shannon sampling series for signals in $\mathcal{P} \mathcal{W}_{\pi}^{1}$ was repeated and, furthermore, also made for the mean-square convergence of I-processes. Disregarding the differences between the symmetric and the nonsymmetric Shannon sampling series, Brown claims in the introduction to [12] that

$$
\mathbb{E}\left|X(t)-\sum_{k=-M}^{N} X(k) \frac{\sin (\pi(t-k))}{\pi(t-k)}\right|^{2}
$$

converges to zero for all I-processes $X$. In Theorem 1, we will see that this is not true.
As in [20], the theorems in [12] concerning the upper bounds on the mean-square approximation error are correct, because in the theorems additional assumptions on the I-processes are made. One assumption is that $S$ is continuous, and the other is that a guard band is present. So, for a restricted class of I-processes we have the mean-square convergence of the nonsymmetric Shannon sampling series. In Theorem 2 we will completely characterize this subclass, i.e., the I-processes for which the "claim" in the introduction to [12] is true, by giving a necessary and sufficient condition for the convergence of (5).

\section{MAIN RESUlts}

It is tempting to try to reduce the analysis of the nonsymmetric sampling series to the analysis of the symmetric sampling series by splitting up the nonsymmetric sampling series into a symmetric series and a remaining term according to

$$
\begin{aligned}
\sum_{k=-M}^{N} X(k) \frac{\sin (\pi(t-k))}{\pi(t-k)}= & \sum_{k=-M}^{M} X(k) \frac{\sin (\pi(t-k))}{\pi(t-k)} \\
& +\sum_{k=M+1}^{N} X(k) \frac{\sin (\pi(t-k))}{\pi(t-k)}
\end{aligned}
$$

where we assumed without loss of generality that $N>M$, and by bounding the contribution of the remaining term to the mean-square approximation error. However, in Theorem 1 we will see that it is not always possible to bound

$$
\mathbb{E}\left|\sum_{k=M+1}^{N} X(k) \frac{\sin (\pi(t-k))}{\pi(t-k)}\right|^{2}
$$

independently of $M$ and $N$, because there are I-processes $X$ such that, for every $M \in \mathbb{N}$ and all $t \in \mathbb{R} \backslash \mathbb{Z}$, we have

$$
\lim _{N \rightarrow \infty} \mathbb{E}\left|\sum_{k=M+1}^{N} X(k) \frac{\sin (\pi(t-k))}{\pi(t-k)}\right|^{2}=\infty .
$$

In Theorem 1, we analyze the boundedness of the mean-square approximation error of the symmetric and the nonsymmetric sapling series and completely characterize the I-processes for which we have the divergence (6) of the nonsymmetric sampling series.

Theorem 1: Let $X$ be an I-process. Then we have

$$
\sup _{N, M \in \mathbb{N}}\left(\max _{t \in \mathbb{R}} \mathbb{E}\left|X(t)-\sum_{k=-M}^{N} X(k) \frac{\sin (\pi(t-k))}{\pi(t-k)}\right|^{2}\right)<\infty
$$

if and only if the power spectral density $S$ fulfills

$$
\int_{-\pi}^{\pi} S(\omega)\left|\log \left(2 \cos \left(\frac{\omega}{2}\right)\right)\right|^{2} \mathrm{~d} \omega<\infty .
$$

Moreover, we have

$$
\limsup _{N, M \rightarrow \infty} \mathbb{E}\left|X(t)-\sum_{k=-M}^{N} X(k) \frac{\sin (\pi(t-k))}{\pi(t-k)}\right|^{2}=\infty
$$


for all $t \in \mathbb{R} \backslash \mathbb{Z}$ if and only if (8) is not fulfilled, and

$$
\lim _{N \rightarrow \infty}\left(\max _{t \in \mathbb{R}} \mathbb{E}\left|X(t)-\sum_{k=-N}^{N} X(k) \frac{\sin (\pi(t-k))}{\pi(t-k)}\right|^{2}\right)=\infty
$$

if and only if (8) is not fulfilled.

Theorem 1 only makes an assertion about the boundedness of the mean-square approximation error. But even if this error is bounded, we cannot conclude the convergence of the nonsymmetric sampling series. The convergence of the nonsymmetric sampling series is treated in the next theorem.

Theorem 2: Let $X$ be an I-process. Then for all $0<T<\infty$, we have

$$
\begin{aligned}
\lim _{N, M \rightarrow \infty}\left(\max _{t \in[-T, T]} \mathbb{E} \mid X(t)\right. & \\
& \left.\quad-\left.\sum_{k=-M}^{N} X(k) \frac{\sin (\pi(t-k))}{\pi(t-k)}\right|^{2}\right)=0
\end{aligned}
$$

if and only if the power spectral density $S$ fulfills (8).

Theorem 2 gives a necessary and sufficient condition for the local uniform convergence in the mean-square sense of the nonsymmetric sampling series. That is, the nonsymmetric sampling series is locally uniformly convergent in the mean-square sense if and only if the condition (8) on the power spectral density is fulfilled. This highlights the difference between the nonsymmetric and the symmetric Shannon sampling series, where we always have-according to (4) — local uniform convergence, regardless of the power spectral density $S$.

Remark 1: By setting $M=N,(7)$ also implies the global boundedness of the mean-square approximation error of the symmetric sampling series. Thus, if (8) is fulfilled, we have

$$
\sup _{N \in \mathbb{N}} \max _{t \in \mathbb{R}} \mathbb{E}\left|X(t)-\sum_{k=-N}^{N} X(k) \frac{\sin (\pi(t-k))}{\pi(t-k)}\right|^{2}<\infty .
$$

Remark 2: The symmetric sampling series is a special case of the nonsymmetric sampling series with $M=N$. Thus, some properties of the symmetric sampling series can be inferred from the properties of the nonsymmetric sampling series. For example, according to the definition of the convergence of the nonsymmetric sampling series, the convergence of the symmetric sampling series follows directly from the convergence of the nonsymmetric sampling series. However, the divergence of the nonsymmetric sampling series does not imply the divergence of the symmetric sampling series. This is because $M$ and $N$ can tend independently to infinity in the nonsymmetric sampling series. In Section IV, we will give an example of a power spectral density for which the mean-square approximation error of the nonsymmetric sampling series diverges for all $t \in \mathbb{R} \backslash \mathbb{Z}$, whereas the mean-square approximation error of the symmetric sampling series converges to zero uniformly on all compact subsets of $\mathbb{R}$.
Remark 3: Since condition (8) is a necessary and sufficient condition for the global boundedness of the mean-square approximation error of the symmetric as well as the nonsymmetric sampling series, we have completely characterized the global boundedness of the mean-square approximation error of the symmetric and the nonsymmetric sampling series.

Remark 4: It is interesting to note that in (10), we do not have a limit superior, as is the case for many divergence results for Fourier series, but a limit. Thus, the existence of a convergent subsequence is precluded.

Remark 5: For the nonsymmetric Shannon sampling series, we have the special situation that convergence in mean-square sense for a fixed $t \in \mathbb{R} \backslash \mathbb{Z}$ implies convergence in mean-square sense for all $t \in \mathbb{R}$ and uniform convergence on compact subsets of $\mathbb{R}$.

Remark 6: Of course we can neither expect the symmetric nor the nonsymmetric sampling series to be globally uniformly convergent in the mean-square sense. This is because $X$ is an I-process and hence

$$
\mathbb{E}|X(t)|^{2}=\frac{1}{2 \pi} \int_{-\pi}^{\pi} S(\omega) \mathrm{d} \omega=C_{1}
$$

where $C_{1}$ is a constant ${ }^{1}$ that is independent of $t \in \mathbb{R}$. Thus, for all $M, N \in \mathbb{N}$, we have

$$
\begin{aligned}
\lim _{t \rightarrow \infty} \mathbb{E}\left|X(t)-\sum_{k=-M}^{N} X(k) \frac{\sin (\pi(t-k))}{\pi(t-k)}\right|^{2} & \\
& =\frac{1}{2 \pi} \int_{-\pi}^{\pi} S(\omega) \mathrm{d} \omega
\end{aligned}
$$

and consequently

$$
\begin{aligned}
\max _{t \in \mathbb{P}} \mathbb{E}\left|X(t)-\sum_{k=-M}^{N} X(k) \frac{\sin (\pi(t-k))}{\pi(t-k)}\right|^{2} & \\
& \geq \frac{1}{2 \pi} \int_{-\pi}^{\pi} S(\omega) \mathrm{d} \omega .
\end{aligned}
$$

In order to prove the theorems, we need Lemmas 1 and 2, the proofs of which are given in the Appendix.

Lemma 1: There exists a constant $C_{2}$ such that

$$
\left|\sum_{k=M}^{N} \mathrm{e}^{i \omega k} \frac{\sin (\pi(t-k))}{\pi(t-k)}\right| \leq \frac{2}{\pi}\left|\log \left(2 \cos \left(\frac{\omega}{2}\right)\right)\right|+C_{2}
$$

for all $t \in \mathbb{R}, M, N \in \mathbb{Z}, M \leq N$, and $\omega \in[-\pi, \pi]$.

Lemma 2: Given any $\epsilon>0,0<\delta<\pi$, and $T>0$, there exist two natural numbers $N_{0}=N_{0}(\epsilon, \delta, T)$ and $M_{0}=$ $M_{0}(\epsilon, \delta, T)$ such that for all $N \geq N_{0}, M \geq M_{0}$

$$
\max _{t \in[-T, T]} \max _{|\omega| \leq \pi-\delta}\left|\mathrm{e}^{i \omega t}-\sum_{k=-M}^{N} \mathrm{e}^{i \omega k} \frac{\sin (\pi(t-k))}{\pi(t-k)}\right|<\epsilon .
$$

Now we are in the position to prove Theorem 1.

${ }^{1}$ Throughout the paper, we have to use many constants. To keep the notation as simple as possible, we denote by $C_{1}, C_{2}, \ldots$ nonnegative constants, unless otherwise stated. 
Proof of Theorem 1: Part I: First, we prove the " $\Leftarrow$ " direction of the first "if and only if" assertion, i.e., (8) $\Rightarrow(7)$. Suppose (8) is true, and let $t \in \mathbb{R}$ and $N, M \in \mathbb{N}$ be arbitrary but fixed. Using the abbreviation

$$
q_{M, N}(t, \omega):=\sum_{k=M}^{N} \mathrm{e}^{i \omega k} \frac{\sin (\pi(t-k))}{\pi(t-k)}
$$

we have

$$
\begin{aligned}
&\left(\mathbb{E}\left|X(t)-\sum_{k=-M}^{N} X(k) \frac{\sin (\pi(t-k))}{\pi(t-k)}\right|^{2}\right)^{\frac{1}{2}} \\
&=\left(\frac{1}{2 \pi} \int_{-\pi}^{\pi} S(\omega)\left|\mathrm{e}^{i \omega t}-q_{-M, N}(t, \omega)\right|^{2} \mathrm{~d} \omega\right)^{\frac{1}{2}} \\
& \leq\left(\frac{1}{2 \pi} \int_{-\pi}^{\pi} S(\omega) \mathrm{d} \omega\right)^{\frac{1}{2}} \\
& \quad+\left(\frac{1}{2 \pi} \int_{-\pi}^{\pi} S(\omega)\left|q_{-M, N}(t, \omega)\right|^{2} \mathrm{~d} \omega\right)^{\frac{1}{2}} \\
& \leq\left(1+C_{2}\right)\left(\frac{1}{2 \pi} \int_{-\pi}^{\pi} S(\omega) \mathrm{d} \omega\right)^{\frac{1}{2}} \\
&+\frac{2}{\pi}\left(\frac{1}{2 \pi} \int_{-\pi}^{\pi} S(\omega)\left|\log \left(2 \cos \left(\frac{\omega}{2}\right)\right)\right|^{2} \mathrm{~d} \omega\right)^{\frac{1}{2}} \\
& \leq C_{3} .
\end{aligned}
$$

The second to last inequality follows from Lemma 1 and the last inequality from the fact that $S \in L^{1}[-\pi, \pi]$ and the assumption (8). Since $t \in \mathbb{R}$ and $N, M \in \mathbb{N}$ were arbitrary, this completes the proof of the " $\Leftarrow$ " direction of the first if and only if assertion. Note that the " $\Leftarrow$ " direction of the first "if and only if" assertion also implies the " $\Rightarrow$ " direction of the second "if and only if" assertion.

Part II: Next, we prove the " $\Leftarrow$ " direction of the second "if and only if" assertion, i.e., (not (8)) $\Rightarrow(9)$. Note that this direction of the second "if and only if" assertion also implies the " $\Rightarrow$ " direction of the first "if and only if" assertion. Suppose that (8) is not fulfilled, i.e., that

$$
\int_{-\pi}^{\pi} S(\omega)\left|\log \left(2 \cos \left(\frac{\omega}{2}\right)\right)\right|^{2} \mathrm{~d} \omega=\infty
$$

Let $t, t_{1} \in \mathbb{R} \backslash \mathbb{Z}$ and $N \in \mathbb{N}$ be arbitrary but fixed. We have

$$
\begin{aligned}
\left(\mathbb{E}\left|X(t)-\sum_{k=1}^{N} X(k) \frac{\sin (\pi(t-k))}{\pi(t-k)}\right|^{2}\right)^{\frac{1}{2}} \\
\geq\left(\frac{1}{2 \pi} \int_{-\pi}^{\pi} S(\omega)\left|q_{1, N}(t, \omega)\right|^{2} \mathrm{~d} \omega\right)^{\frac{1}{2}} \\
\quad-\left(\frac{1}{2 \pi} \int_{-\pi}^{\pi} S(\omega) \mathrm{d} \omega\right)^{\frac{1}{2}}
\end{aligned}
$$

where $q_{M, N}(t, \omega)$ is the abbreviation that was introduced in (11). Further, since

$$
\begin{aligned}
& \left|\frac{q_{1, N}(t, \omega)}{\sin (t)}-\frac{q_{1, N}\left(t_{1}, \omega\right)}{\sin \left(t_{1}\right)}\right| \\
& \quad=\frac{1}{\pi}\left|\sum_{k=1}^{N} \mathrm{e}^{i \omega k} \frac{(-1)^{k}\left(t_{1}-t\right)}{(t-k)\left(t_{1}-k\right)}\right| \\
& \quad \leq \frac{\left|t_{1}-t\right|}{\pi} \sum_{k=1}^{\infty} \frac{1}{|t-k|\left|t_{1}-k\right|} \\
& \quad=C_{4}\left(t, t_{1}\right)
\end{aligned}
$$

with a constant $C_{4}\left(t, t_{1}\right)$ that depends only on $t$ and $t_{1}$, we obtain

$$
\begin{aligned}
& \frac{1}{\sin (t)}\left(\int_{-\pi}^{\pi} S(\omega)\left|q_{1, N}(t, \omega)\right|^{2} \mathrm{~d} \omega\right)^{\frac{1}{2}} \\
& \geq\left(\int_{-\pi}^{\pi} S(\omega)\left|\frac{q_{1, N}\left(t_{1}, \omega\right)}{\sin \left(t_{1}\right)}\right|^{2} \mathrm{~d} \omega\right)^{\frac{1}{2}} \\
& \quad-\left(\int_{-\pi}^{\pi} S(\omega)\left|\frac{q_{1, N}(t, \omega)}{\sin (t)}-\frac{q_{1, N}\left(t_{1}, \omega\right)}{\sin \left(t_{1}\right)}\right|^{2} \mathrm{~d} \omega\right)^{\frac{1}{2}} \\
& =\frac{1}{\sin \left(t_{1}\right)}\left(\int_{-\pi}^{\pi} S(\omega)\left|q_{1, N}\left(t_{1}, \omega\right)\right|^{2} \mathrm{~d} \omega\right)^{\frac{1}{2}} \\
& \quad-C_{4}\left(t, t_{1}\right)\left(\int_{-\pi}^{\pi} S(\omega) \mathrm{d} \omega\right)^{\frac{1}{2}} .
\end{aligned}
$$

From (13) and (14), we see that it is enough to show that

$$
\liminf _{N \rightarrow \infty}\left(\int_{-\pi}^{\pi} S(\omega)\left|q_{1, N}\left(t_{1}, \omega\right)\right|^{2} \mathrm{~d} \omega\right)^{\frac{1}{2}}=\infty
$$

for some $t_{1} \in \mathbb{R} \backslash \mathbb{Z}$ in order to prove part II of the proof. We choose $t_{1}=1 / 2$. For $0<\delta<\pi$, we have

$$
\begin{aligned}
& \left(\int_{-\pi}^{\pi} S(\omega)\left|q_{1, N}\left(t_{1}, \omega\right)\right|^{2} \mathrm{~d} \omega\right)^{\frac{1}{2}} \\
& =\left(\int_{-\pi}^{\pi} S(\omega)\left|\sum_{k=1}^{N} \mathrm{e}^{i \omega k} \frac{(-1)^{k}}{\pi\left(k-\frac{1}{2}\right)}\right|^{2} \mathrm{~d} \omega\right)^{\frac{1}{2}} \\
& \geq\left(\int_{-\pi+\delta}^{\pi-\delta} S(\omega)\left|\sum_{k=1}^{N} \mathrm{e}^{i \omega k} \frac{(-1)^{k}}{\pi k}\right|^{2} \mathrm{~d} \omega\right)^{\frac{1}{2}}-C_{5}
\end{aligned}
$$

because

$$
\begin{aligned}
& \left|\sum_{k=1}^{N} \mathrm{e}^{i \omega k} \frac{(-1)^{k}}{\pi k}\right| \\
& \quad \leq\left|\sum_{k=1}^{N} \frac{\mathrm{e}^{i \omega k}(-1)^{k}}{\pi}\left(\frac{1}{k}-\frac{1}{k-\frac{1}{2}}\right)\right|+\left|\sum_{k=1}^{N} \frac{\mathrm{e}^{i \omega k}(-1)^{k}}{\pi\left(k-\frac{1}{2}\right)}\right| \\
& \quad=\left|\sum_{k=1}^{N} \frac{\mathrm{e}^{i \omega k}(-1)^{k}}{2 \pi} \frac{1}{k\left(k-\frac{1}{2}\right)}\right|+\left|\sum_{k=1}^{N} \frac{\mathrm{e}^{i \omega k}(-1)^{k}}{\pi\left(k-\frac{1}{2}\right)}\right| \\
& \quad \leq C_{6}+\left|\sum_{k=1}^{N} \mathrm{e}^{i \omega k} \frac{(-1)^{k}}{\pi\left(k-\frac{1}{2}\right)}\right|
\end{aligned}
$$


for all $\omega \in[-\pi, \pi]$. It follows that:

$$
\begin{aligned}
& \liminf _{N \rightarrow \infty}\left(\int_{-\pi}^{\pi} S(\omega)\left|q_{1, N}\left(t_{1}, \omega\right)\right|^{2} \mathrm{~d} \omega\right)^{\frac{1}{2}} \\
& \quad \geq \liminf _{N \rightarrow \infty}\left(\int_{-\pi+\delta}^{\pi-\delta} S(\omega)\left|\sum_{k=1}^{N} \mathrm{e}^{i \omega k} \frac{(-1)^{k}}{\pi k}\right|^{2} \mathrm{~d} \omega\right)^{\frac{1}{2}}-C_{5} \\
& \quad \geq \frac{1}{\pi}\left(\int_{-\pi+\delta}^{\pi-\delta} S(\omega)\left|\log \left(2 \cos \left(\frac{\omega}{2}\right)\right)\right|^{2} \mathrm{~d} \omega\right)^{\frac{1}{2}}-C_{7}
\end{aligned}
$$

where we used Fatou's Lemma [27, p. 23] and

$$
\begin{aligned}
\liminf _{N \rightarrow \infty}\left|\sum_{k=1}^{N} \mathrm{e}^{i \omega k} \frac{(-1)^{k}}{\pi k}\right|^{2} & \\
& \geq \frac{1}{\pi^{2}}\left(\left|\log \left(2 \cos \left(\frac{\omega}{2}\right)\right)\right|-\frac{\pi}{2}\right)^{2}
\end{aligned}
$$

for $|\omega|<\pi$. Equation (17) follows from

$$
\begin{aligned}
-\sum_{k=1}^{\infty} \frac{\mathrm{e}^{i \omega k}}{k}=\frac{1}{2} \log (2- & 2 \cos (\omega)) \\
& +\frac{i}{2} \operatorname{sgn}(\omega)(|\omega|-\pi)=\log \left(1-\mathrm{e}^{i \omega}\right)
\end{aligned}
$$

for $\omega \in[-\pi, \pi], \omega \neq 0$, which is a consequence of $[28,1.441]$, the identity

$$
\sin \left(\frac{\omega+\pi}{2}\right)=\cos \left(\frac{\omega}{2}\right)
$$

and the fact that

$$
\left|\log \left(1-\mathrm{e}^{i \omega}\right)\right| \geq\left|\log \left(2\left|\sin \left(\frac{\omega}{2}\right)\right|\right)\right|-\frac{\pi}{2}
$$

for $\omega \in[-\pi, \pi], \omega \neq 0$. Due to our assumption (12), and since (16) is valid for all $0<\delta<\pi$, we have proved (15), which completes part II of the proof.

Part III: Next, we prove the third "if and only if" assertion. We start with the " $\Rightarrow$ " direction. By setting $M=N,(7)$ also implies the global boundedness of the mean-square approximation error of the symmetric sampling series. Thus, if (8) is fulfilled we have

$$
\sup _{N \in \mathbb{N}}\left(\max _{t \in \mathbb{R}} \mathbb{E}\left|X(t)-\sum_{k=-N}^{N} X(k) \frac{\sin (\pi(t-k))}{\pi(t-k)}\right|^{2}\right)<\infty .
$$

It remains to prove the " $\Leftarrow$ " direction of the third "if and only if" assertion, i.e., (not (8)) $\Rightarrow(10)$. This proof is done analogously to the proof of the " $\Leftarrow$ " direction of the second "if and only if" assertion, i.e., part II of the proof, and thus is omitted.

Next we prove Theorem 2.

Proof of Theorem 2: “ $\Leftarrow$ ": Let $T \in \mathbb{R}$ with $0<T<\infty$ be arbitrary but fixed. Since (8) is fulfilled, for any $\epsilon>0$ there exists a $0<\delta_{0}<\pi$ such that

$$
\frac{1}{2 \pi} \int_{\pi-\delta_{0} \leq|\omega| \leq \pi} S(\omega)\left|\log \left(2 \cos \left(\frac{\omega}{2}\right)\right)\right|^{2} \mathrm{~d} \omega<\epsilon^{2}
$$

and

$$
\left|\log \left(2 \cos \left(\frac{\omega}{2}\right)\right)\right|>1
$$

for all $|\omega| \in\left[\pi-\delta_{0}, \pi\right]$. Now, let $\delta_{0}$ be fixed. Then for $t \in$ $[-T, T]$, we have

$$
\begin{aligned}
\left(\mathbb{E}\left|X(t)-\sum_{k=-M}^{N} X(k) \frac{\sin (\pi(t-k))}{\pi(t-k)}\right|^{2}\right)^{\frac{1}{2}} \\
=\left(\frac{1}{2 \pi} \int_{|\omega| \leq \pi-\delta_{0}} S(\omega)\left|\mathrm{e}^{i \omega t}-q_{-M, N}(t, \omega)\right|^{2} \mathrm{~d} \omega\right)^{\frac{1}{2}} \\
\quad+\left(\frac{1}{2 \pi} \int_{\pi-\delta_{0} \leq|\omega| \leq \pi} S(\omega)\left|\mathrm{e}^{i \omega t}-q_{-M, N}(t, \omega)\right|^{2} \mathrm{~d} \omega\right)^{\frac{1}{2}}
\end{aligned}
$$

where $q_{M, N}(t, \omega)$ is the abbreviation that was introduced in (11). The first term on the right hand side of (20) can be upper bounded as follows: By Lemma 2 we know that, given any $\epsilon>0$, there are two constants $N_{0}=N_{0}(\epsilon, \delta, T)$ and $M_{0}=$ $M_{0}(\epsilon, \delta, T)$ such that for all $N \geq N_{0}$ and $M \geq M_{0}$

$$
\max _{t \in[-T, T]} \max _{|\omega| \leq \pi-\delta_{0}}\left|\mathrm{e}^{i \omega t}-q_{-M, N}(t, \omega)\right|<\epsilon .
$$

It follows that

$$
\left(\frac{1}{2 \pi} \int_{|\omega| \leq \pi-\delta_{0}} S(\omega)\left|\mathrm{e}^{i \omega t}-q_{-M, N}(t, \omega)\right|^{2} \mathrm{~d} \omega\right)^{\frac{1}{2}} \leq C_{8} \epsilon .
$$

The second term on the right-hand side of (20) gives

$$
\begin{aligned}
& \left(\frac{1}{2 \pi} \int_{\pi-\delta_{0} \leq|\omega| \leq \pi} S(\omega)\left|\mathrm{e}^{i \omega t}-q_{-M, N}(t, \omega)\right|^{2} \mathrm{~d} \omega\right)^{\frac{1}{2}} \\
& \leq\left(\frac{1}{2 \pi} \int_{\pi-\delta_{0} \leq|\omega| \leq \pi} S(\omega) \mathrm{d} \omega\right)^{\frac{1}{2}} \\
& \quad+\left(\frac{1}{2 \pi} \int_{\pi-\delta_{0} \leq|\omega| \leq \pi} S(\omega)\left|q_{-M, N}(t, \omega)\right|^{2} \mathrm{~d} \omega\right)^{\frac{1}{2}} .
\end{aligned}
$$

The first term is smaller than $\epsilon$, and for the second term we obtain

$$
\begin{aligned}
& \left(\frac{1}{2 \pi} \int_{\pi-\delta_{0} \leq|\omega| \leq \pi} S(\omega)\left|q_{-M, N}(t, \omega)\right|^{2} \mathrm{~d} \omega\right)^{\frac{1}{2}} \\
& \leq\left(\frac{1}{2 \pi} \int_{\pi-\delta_{0} \leq|\omega| \leq \pi} S(\omega)\right. \\
& \left.\quad \times\left(\frac{2}{\pi}\left|\log \left(2 \cos \left(\frac{\omega}{2}\right)\right)\right|+C_{2}\right)^{2} \mathrm{~d} \omega\right)^{\frac{1}{2}} \\
& \leq C_{9} \epsilon
\end{aligned}
$$


were we used Lemma 1 in the second to last inequality and (19) in the last inequality. Combining all partial results, we get

$$
\mathbb{E}\left|X(t)-\sum_{k=-M}^{N} X(k) \frac{\sin (\pi(t-k))}{\pi(t-k)}\right|^{2} \leq\left(C_{8}+1+C_{9}\right)^{2} \epsilon^{2}
$$

for all $N \geq N_{0}, M \geq M_{0}$, and $t \in[-T, T]$. Since $\epsilon$ was arbitrary, this part of the proof is complete. " $\Rightarrow$ ": This direction follows from the second "if and only if" assertion of Theorem 1.

\section{DISCUSSION}

As we have seen in Theorems 1 and 2, is important to know when (8) is fulfilled and when it is not. There are several special cases where (8) is true. One important case is when $S$ is continuous and another is the case when $S \in L^{p}[-\pi, \pi], 1<p \leq \infty$. According to Hölder's inequality, we have for $1<p \leq \infty$, $1 / p+1 / q=1$

$$
\begin{aligned}
\int_{-\pi}^{\pi} & S(\omega)\left|\log \left(2 \cos \left(\frac{\omega}{2}\right)\right)\right|^{2} \mathrm{~d} \omega \\
\leq & \left(\int_{-\pi}^{\pi}(S(\omega))^{p} \mathrm{~d} \omega\right)^{1 / p} \\
& \times\left(\int_{-\pi}^{\pi}\left|\log \left(2 \cos \left(\frac{\omega}{2}\right)\right)\right|^{2 q} \mathrm{~d} \omega\right)^{1 / q} .
\end{aligned}
$$

In both of the cases- $S$ is continuous or $S \in L^{p}[-\pi, \pi]$ - the first term on the right-hand side of (22) is finite. It remains to show that the second term on the right hand side of (22) is finite. For $r \geq 1$, we have

$$
\begin{aligned}
\int_{-\pi}^{\pi} & \left|\log \left(2 \cos \left(\frac{\omega}{2}\right)\right)\right|^{r} \mathrm{~d} \omega \\
= & \int_{-\frac{3 \pi}{4}}^{\frac{3 \pi}{4}}\left|\log \left(2 \cos \left(\frac{\omega}{2}\right)\right)\right|^{r} \mathrm{~d} \omega \\
& +2 \int_{\frac{3 \pi}{4}}^{\pi}\left[-\log \left(2 \cos \left(\frac{\omega}{2}\right)\right)\right]^{r} \mathrm{~d} \omega .
\end{aligned}
$$

Since $|\log (2 \cos (\omega / 2))|^{r}$ is continuous on $[-3 \pi / 4,3 \pi / 4]$, the first summand on the right-hand side of (23) is finite. Furthermore, since $\cos (\omega / 2) \geq 1-\omega / \pi$ for all $\omega \in[3 \pi / 4, \pi]$, it follows that

$$
\begin{aligned}
& 2 \int_{\frac{3 \pi}{4}}^{\pi}\left[-\log \left(2 \cos \left(\frac{\omega}{2}\right)\right)\right]^{r} \mathrm{~d} \omega \\
& \quad \leq 2 \int_{\frac{3 \pi}{4}}^{\pi}\left[-\log \left(2-\frac{2 \omega}{\pi}\right)\right]^{r} \mathrm{~d} \omega \\
& \quad=\int_{\log (2)}^{\infty} u^{r} \mathrm{e}^{-u} \mathrm{~d} u<\infty
\end{aligned}
$$

where we used the substitution $u=-\log (2-2 \omega / \pi)$ in the second to last line. So we have (8) if $S$ is continuous or if $S \in$ $L^{p}[-\pi, \pi], 1<p \leq \infty$.
On the other hand there are power spectral densities $S \in$ $L^{1}[-\pi, \pi]$ for which (8) is not fulfilled. One example is given by

$$
S_{1}(\omega)=\frac{1}{(\omega+\pi)\left(\log \left(\frac{4 \pi}{\omega+\pi}\right)\right)^{2}} .
$$

The short calculation

$$
\begin{aligned}
\int_{-\pi}^{\pi}\left|S_{1}(\omega)\right| \mathrm{d} \omega & =\int_{0}^{2 \pi} \frac{1}{\omega\left(\log \left(\frac{4 \pi}{\omega}\right)\right)^{2}} \mathrm{~d} \omega \\
& =\int_{\log (2)}^{\infty} \frac{1}{u^{2}} \mathrm{~d} u=\frac{1}{\log (2)}<\infty
\end{aligned}
$$

shows that $S_{1} \in L^{1}[-\pi, \pi]$. Next, we show that (8) is not fulfilled for $S_{1}$. We have

$$
\begin{gathered}
\int_{-\pi}^{\pi} S_{1}(\omega)\left|\log \left(2 \cos \left(\frac{\omega}{2}\right)\right)\right|^{2} \mathrm{~d} \omega \\
=\int_{-\pi}^{-\frac{3 \pi}{4}} \frac{\left|\log \left(2 \cos \left(\frac{\omega}{2}\right)\right)\right|^{2}}{(\omega+\pi)\left(\log \left(\frac{4 \pi}{\omega+\pi}\right)\right)^{2}} \mathrm{~d} \omega \\
\quad+\int_{-\frac{3 \pi}{4}}^{\pi} \frac{\left|\log \left(2 \cos \left(\frac{\omega}{2}\right)\right)\right|^{2}}{(\omega+\pi)\left(\log \left(\frac{4 \pi}{\omega+\pi}\right)\right)^{2}} \mathrm{~d} \omega .
\end{gathered}
$$

The second integral is finite because

$$
\begin{aligned}
& \int_{-\frac{3 \pi}{4}}^{\pi} \frac{\left|\log \left(2 \cos \left(\frac{\omega}{2}\right)\right)\right|^{2}}{(\omega+\pi)\left(\log \left(\frac{4 \pi}{\omega+\pi}\right)\right)^{2}} \mathrm{~d} \omega \\
& \leq \int_{-\frac{3 \pi}{4}}^{\pi} \frac{\left|\log \left(2 \cos \left(\frac{\omega}{2}\right)\right)\right|^{2}}{\frac{\pi}{4}(\log (2))^{2}} \mathrm{~d} \omega<\infty
\end{aligned}
$$

according to (24). Furthermore, since

$$
\begin{aligned}
& \int_{-\pi}^{-\frac{3 \pi}{4}} \frac{\left|\log \left(2 \cos \left(\frac{\omega}{2}\right)\right)\right|^{2}}{(\omega+\pi)\left(\log \left(\frac{4 \pi}{\omega+\pi}\right)\right)^{2}} \mathrm{~d} \omega \\
& \quad \geq \int_{-\pi}^{-\frac{3 \pi}{4}} \frac{|\log (\omega+\pi)|^{2}}{(\omega+\pi)\left(\log \left(\frac{4 \pi}{\omega+\pi}\right)\right)^{2}} \mathrm{~d} \omega \\
& \quad=\int_{\log (16)}^{\infty} \frac{(u-\log (4 \pi))^{2}}{u^{2}} \mathrm{~d} \omega \\
& \geq \int_{\log (16)}^{\infty} \frac{u-2 \log (4 \pi)}{u} \mathrm{~d} \omega \\
& \geq[\log (16)-2 \log (4 \pi)] \int_{\log (16)}^{\infty} \frac{1}{u} \mathrm{~d} \omega \\
& =\infty
\end{aligned}
$$

where we used the inequality $\cos (\omega / 2) \leq(\omega+\pi) / 2, \omega \in$ $[-\pi,-3 \pi / 4]$, and the substitution $u=\log (4 \pi /(\omega+\pi))$, we obtain that

$$
\int_{-\pi}^{\pi} S_{1}(\omega)\left|\log \left(2 \cos \left(\frac{\omega}{2}\right)\right)\right|^{2} \mathrm{~d} \omega=\infty .
$$

This shows that, in contrast to the claims in [12], there is a significant difference in the mean-square convergence behavior of the Shannon sampling series for I-processes with $S \in L^{1}[-\pi, \pi]$ and I-processes with $S \in L^{2}[-\pi, \pi]$. 


\section{CONCLUSION}

We have completely characterized the I-processes for which the mean-square approximation error of the nonsymmetric and the symmetric sampling series is globally bounded, by providing the condition (8) on the power spectral density, which is sufficient as well as necessary for the boundedness. Further, it turned out that the same condition is sufficient and necessary for the local uniform convergence in the mean-square sense of the nonsymmetric sampling series. The results show that there is a significant difference in the convergence behavior of the symmetric and the nonsymmetric sampling series. For example, for an I-process $X$ whose power spectral density does not satisfy (8), we have

$$
\limsup _{N, M \rightarrow \infty} \mathbb{E}\left|X(t)-\sum_{k=-M}^{N} X(k) \frac{\sin (\pi(t-k))}{\pi(t-k)}\right|^{2}=\infty
$$

for all $t \in \mathbb{R} \backslash \mathbb{Z}$, whereas for all I-processes, irrespectively of (8), we have

$\lim _{N \rightarrow \infty}\left(\max _{t \in[-T, T]} \mathbb{E}\left|X(t)-\sum_{k=-N}^{N} X(k) \frac{\sin (\pi(t-k))}{\pi(t-k)}\right|^{2}\right)$

for all $0<T<\infty$.

A slightly more general problem than the approximation of I-processes $X$ by sampling series is the approximation of output processes $T X$ of energy stable linear time-invariant (LTI) systems $T$ by sampling series, according to

$$
\mathbb{E}\left|(T X)(t)-\sum_{k=-N}^{N} X(k) h_{T}(t-k)\right|^{2}
$$

where $h_{T}=T \sin (\pi \cdot) /(\pi \cdot)$. The symmetric sampling series in this paper can be seen as a special case of the series in (25), where the system $T$ is the identity operator. For this special case, we characterized the I-processes for which (25) is globally bounded as $N$ tends to infinity, and found that there are I-processes $X$ for which the supremum of (25) over $\mathbb{R}$ diverges. In [29], we have characterized the systems $T$ for which (25) converges pointwise to zero for all I-processes $X$. An interesting open problem is to characterize the I-processes $X$ for which (25) converges pointwise to zero for all energy stable LTI systems $T$.

\section{APPENDIX}

\section{A. Proof of Lemma 1}

The proof of Lemma 1 requires two more lemmas, namely Lemma 3 and Lemma 4.

Lemma 3: There exists a positive constant $C_{10}$, such that

$$
\left|\sum_{k=1}^{N} \frac{\mathrm{e}^{i k \omega}}{k}\right| \leq\left|\log \left(2\left|\sin \left(\frac{\omega}{2}\right)\right|\right)\right|+C_{10}
$$

for all $N \in \mathbb{N}$ and $\omega \in[-\pi, \pi]$.
Proof of Lemma 3: We analyze $\sum_{k=N}^{M} \frac{\mathrm{e}^{i \omega k}}{k}$ for $\omega \in[-\pi, \pi], \omega \neq 0$, and $N, M \in \mathbb{N}, N<M$, using summation by parts. For $k \geq N$, let

$$
D_{k, N}(\omega):=\sum_{l=N}^{k} \mathrm{e}^{i \omega l} .
$$

Then, using summation by parts, we obtain

$$
\sum_{k=N+1}^{M} \frac{\mathrm{e}^{i \omega k}}{k}=\frac{D_{M, N+1}(\omega)}{M}+\sum_{k=N+1}^{M-1} \frac{D_{k, N+1}(\omega)}{k(k+1)} .
$$

Since

$$
\begin{aligned}
\left|D_{k, N+1}(\omega)\right| & =\left|\sum_{l=N+1}^{k} \mathrm{e}^{i \omega l}\right| \\
& =\left|\frac{1-\mathrm{e}^{i \omega(k-N)}}{1-\mathrm{e}^{i \omega}}\right| \leq \frac{1}{\left|\sin \left(\frac{\omega}{2}\right)\right|}
\end{aligned}
$$

the first term in (26), i.e., $D_{M, N+1}(\omega) / M$, converges to zero for $M \rightarrow \infty$. Additionally, for $M>N+1$, we have

$$
\begin{aligned}
\sum_{k=N+1}^{M-1} \frac{\left|D_{k, N+1}(\omega)\right|}{k(k+1)} & \leq \frac{1}{\left|\sin \left(\frac{\omega}{2}\right)\right|} \sum_{k=N+1}^{M-1} \frac{1}{k(k+1)} \\
& <\frac{1}{\left|\sin \left(\frac{\omega}{2}\right)\right| N} .
\end{aligned}
$$

Thus, the sum in (26) is convergent for $M \rightarrow \infty$, and we obtain

$$
\left|\sum_{k=N+1}^{\infty} \frac{\mathrm{e}^{i \omega k}}{k}\right| \leq \frac{1}{\left|\sin \left(\frac{\omega}{2}\right)\right| N}
$$

for $\omega \in[-\pi, \pi], \omega \neq 0$, and $N \in \mathbb{N}$. Further, since

$$
\sum_{k=1}^{\infty} \frac{\mathrm{e}^{i \omega k}}{k}=\sum_{k=1}^{\infty} \frac{\cos (k \omega)}{k}+i \sum_{k=1}^{\infty} \frac{\sin (k \omega)}{k}
$$

it follows from $[28,1.441]$ that

$$
\begin{aligned}
-\sum_{k=1}^{\infty} \frac{\mathrm{e}^{i \omega k}}{k} & =\frac{1}{2} \log (2-2 \cos (\omega))+\frac{i}{2} \operatorname{sgn}(\omega)(|\omega|-\pi) \\
& =\log \left(1-\mathrm{e}^{i \omega}\right)
\end{aligned}
$$

for $\omega \in[-\pi, \pi], \omega \neq 0$. Thus, we have

$$
\begin{aligned}
\left|\log \left(1-\mathrm{e}^{i \omega}\right)+\sum_{k=1}^{N} \frac{\mathrm{e}^{i \omega k}}{k}\right| & =\left|\sum_{k=N+1}^{\infty} \frac{\mathrm{e}^{i \omega k}}{k}\right| \\
& <\frac{1}{\left|\sin \left(\frac{\omega}{2}\right)\right| N}
\end{aligned}
$$

where we used (27) in the last inequality.

Next, we have to distinguish two cases. First, we analyze the case $2 \sin (1 /(2 N)) \leq|\omega| \leq \pi$. We have

$$
\frac{1}{\left|\sin \left(\frac{\omega}{2}\right)\right| N} \leq \frac{\pi}{|\omega| N} \leq \frac{\pi^{2}}{2} \text {. }
$$


Thus, using (28), we obtain

$$
\begin{aligned}
\left|\sum_{k=1}^{N} \frac{\mathrm{e}^{i \omega k}}{k}\right| & \leq \frac{\pi^{2}}{2}+\left|\log \left(1-\mathrm{e}^{i \omega}\right)\right| \\
& \leq \frac{\pi^{2}}{2}+\frac{\pi}{2}+\left|\log \left(2\left|\sin \left(\frac{\omega}{2}\right)\right|\right)\right|
\end{aligned}
$$

for $2 \sin (1 /(2 N)) \leq|\omega| \leq \pi$, because

$$
\left|\log \left(1-\mathrm{e}^{i \omega}\right)\right| \leq\left|\log \left(2\left|\sin \left(\frac{\omega}{2}\right)\right|\right)\right|+\frac{\pi}{2} .
$$

The second case is $0<|\omega| \leq 2 \sin (1 /(2 N))$. We have

$$
\left|\sum_{k=1}^{N} \frac{\mathrm{e}^{i \omega k}}{k}\right| \leq \sum_{k=1}^{N} \frac{1}{k}<1+\log (2 N)
$$

Furthermore, a simple calculation shows that

$$
\log (2 N) \leq \log (2)+\left|\log \left(2\left|\sin \left(\frac{\omega}{2}\right)\right|\right)\right|
$$

and thus, it follows that:

$$
\left|\sum_{k=1}^{N} \frac{\mathrm{e}^{i \omega k}}{k}\right|<1+\log (2)+\left|\log \left(2\left|\sin \left(\frac{\omega}{2}\right)\right|\right)\right|
$$

for $0<|\omega| \leq 2 \sin (1 /(2 N))$. Combining (29) and (30), we have

$$
\left|\sum_{k=1}^{N} \frac{\mathrm{e}^{i \omega k}}{k}\right| \leq \frac{\pi^{2}}{2}+\frac{\pi}{2}+\left|\log \left(2\left|\sin \left(\frac{\omega}{2}\right)\right|\right)\right|
$$

for $\omega \in[-\pi, \pi], \omega \neq 0$, and $N \in \mathbb{N}$. For $\omega=0$, the assertion of the lemma is trivially fulfilled.

Lemma 4: There exists a positive constants $C_{11}$, such that

$$
\left|\sum_{k=M}^{N} \frac{\mathrm{e}^{i \omega k}}{k+\frac{1}{2}}\right| \leq 2\left|\log \left(2\left|\sin \left(\frac{\omega}{2}\right)\right|\right)\right|+C_{11}
$$

for all $M, N \in \mathbb{Z}, M \leq N$, and $\omega \in[-\pi, \pi]$.

Proof of Lemma 4: Let $M, N \in \mathbb{Z}, M \leq N$ arbitrary but fixed. We have

$$
\sum_{k=M}^{N} \frac{\mathrm{e}^{i \omega k}}{k+\frac{1}{2}}-\sum_{\substack{k=M \\ k \neq 0}}^{N} \frac{\mathrm{e}^{i \omega k}}{k}=c_{M, N}-\frac{1}{2} \sum_{\substack{k=M \\ k \neq 0}}^{N} \frac{\mathrm{e}^{i \omega k}}{k\left(k+\frac{1}{2}\right)}
$$

where

$$
c_{M, N}= \begin{cases}2 & M \leq 0 \leq N \\ 0 & \text { otherwise }\end{cases}
$$

and consequently

$$
\left|\sum_{k=M}^{N} \frac{\mathrm{e}^{i \omega k}}{k+\frac{1}{2}}-\sum_{\substack{k=M \\ k \neq 0}}^{N} \frac{\mathrm{e}^{i \omega k}}{k}\right| \leq 2+\frac{1}{2} \sum_{\substack{k=M \\ k \neq 0}}^{N} \frac{1}{k\left(k+\frac{1}{2}\right)} \leq 4 .
$$

We proceed with the convention that an empty sum, i.e., a sum where the upper summation index is less than the lower summation index, is zero. Obviously

$$
\begin{aligned}
\left|\sum_{\substack{k=M \\
k \neq 0}}^{N} \frac{\mathrm{e}^{i \omega k}}{k}\right| & \leq\left|\sum_{k=1}^{-M} \frac{\mathrm{e}^{i \omega k}}{k}\right|+\left|\sum_{k=1}^{N} \frac{\mathrm{e}^{i \omega k}}{k}\right| \\
& \leq 2\left(\left|\log \left(2\left|\sin \left(\frac{\omega}{2}\right)\right|\right)\right|+C_{10}\right)
\end{aligned}
$$

where we used Lemma 3 in the last inequality. Combining (32) and (33), we obtain

$$
\left|\sum_{k=M}^{N} \frac{\mathrm{e}^{i \omega k}}{k+\frac{1}{2}}\right| \leq 2\left(\left|\log \left(2\left|\sin \left(\frac{\omega}{2}\right)\right|\right)\right|+C_{10}\right)+4
$$

which completes the proof.

Now we are in the position to prove Lemma 1

Proof of Lemma 1: Since the assertion of the lemma is obviously true for $t \in \mathbb{Z}$, we can restrict our analysis to the case $t \in \mathbb{R} \backslash \mathbb{Z}$. Let $\lfloor t\rfloor$ denote the largest integer that is smaller or equal to $t$. This implies that $|\lfloor t\rfloor+1 / 2-t|<1 / 2$. Using the abbreviation $q_{M, N}(t, \omega)$ that was introduced in (11), we have

$$
\begin{aligned}
& \left|\frac{q_{M, N}(t, \omega)}{\sin (\pi t)}-\frac{q_{M, N}\left(\lfloor t\rfloor+\frac{1}{2}, \omega\right)}{\sin \left(\pi\left(\lfloor t\rfloor+\frac{1}{2}\right)\right)}\right| \\
& \quad=\left|\frac{1}{\pi} \sum_{k=M}^{N} \mathrm{e}^{i \omega k}(-1)^{k}\left(\frac{1}{t-k}-\frac{1}{\lfloor t\rfloor+\frac{1}{2}-k}\right)\right| \\
& \quad \leq C_{12}+\frac{1}{|t-\lfloor t\rfloor|}+\frac{1}{|t-\lfloor t+1\rfloor|}
\end{aligned}
$$

It follows that

$$
\begin{aligned}
\left|q_{M, N}(t, \omega)\right| \leq & C_{12}|\sin (\pi t)|+\frac{\left|q_{M, N}\left(\lfloor t\rfloor+\frac{1}{2}, \omega\right)\right||\sin (\pi t)|}{\left|\sin \left(\pi\left(\lfloor t\rfloor+\frac{1}{2}\right)\right)\right|} \\
& +\frac{|\sin (\pi t)|}{|t-\lfloor t\rfloor|}+\frac{|\sin (\pi t)|}{|t-\lfloor t+1\rfloor|} \\
\leq & C_{12}+\left|q_{M, N}\left(\lfloor t\rfloor+\frac{1}{2}, \omega\right)\right|+2
\end{aligned}
$$

Furthermore, we have

$$
\begin{aligned}
& \left|q_{M, N}\left(\lfloor t\rfloor+\frac{1}{2}, \omega-\pi\right)\right| \\
& \quad=\left|\frac{1}{\pi} \sum_{k=\lfloor t\rfloor-N}^{\lfloor t\rfloor-M} \frac{\mathrm{e}^{i \omega k}}{k+\frac{1}{2}}\right| \\
& \leq \frac{2}{\pi}\left|\log \left(2\left|\sin \left(\frac{\omega}{2}\right)\right|\right)\right|+C_{13}
\end{aligned}
$$

where the last inequality follows by Lemma 4. Using (18), it follows that:

$$
\left|q_{M, N}\left(\lfloor t\rfloor+\frac{1}{2}, \omega\right)\right| \leq \frac{2}{\pi}\left|\log \left(2\left|\cos \left(\frac{\omega}{2}\right)\right|\right)\right|+C_{13}
$$

and, using (34), that

$$
\left|q_{M, N}(t, \omega)\right| \leq \frac{2}{\pi}\left|\log \left(2\left|\cos \left(\frac{\omega}{2}\right)\right|\right)\right|+C_{2}
$$

which completes the proof. 


\section{B. Proof of Lemma 2}

Proof of Lemma 2: Let $0<\delta<\pi$ and $T>0$ be arbitrary but fixed. From the identity

$$
\frac{\sin (\pi(t-k))}{\pi(t-k)}=\frac{1}{2 \pi} \int_{-\pi}^{\pi} \mathrm{e}^{i \omega t} \mathrm{e}^{-i \omega k} \mathrm{~d} \omega
$$

we see that

$$
\frac{\sin (\pi(t-k))}{\pi(t-k)}, \quad k \in \mathbb{Z}
$$

are the Fourier coefficients of the function $g(\omega, t)$ that is $2 \pi$-periodic in $\omega$ and identical to $\mathrm{e}^{i \omega t}$ for $-\pi \leq \omega<\pi$, where $|t| \leq T$ is a parameter. Now, if

$$
A_{M, N}(\omega, t):=\sum_{k=-M}^{N} \mathrm{e}^{i \omega k} \frac{\sin (\pi(t-k))}{\pi(t-k)}, \quad M, N \in \mathbb{N}
$$

converges for $M, N \rightarrow \infty$ to a function $\tilde{g}(\omega, t)$ for $|t| \leq T$ and $|\omega| \leq \pi-\delta$, then $\tilde{g}(\omega, t)=g(\omega, t)=\mathrm{e}^{i \omega t}$ for $|t| \leq T$ and $|\omega| \leq \pi-\delta$ by the representation theorem for Fourier series and the fact that $\mathrm{e}^{i \omega t}$ is continuous differentiable in $[-\pi+\delta, \pi-\delta]$. It remains to show that $A_{M, N}(\omega, t)$ is uniformly convergent with respect to $t$ and $\omega$ for $|t| \leq T$ and $|\omega| \leq \pi-\delta$ as $M, N \rightarrow \infty$.

In the following analysis, we always assume that $|t| \leq T$ and $|\omega| \leq \pi-\delta$. For $N_{1}, N, M_{1}, M \in \mathbb{N}$ with $N_{1}>N>T$ and $M_{1}>M>T$, we have

$$
\begin{array}{r}
\left|A_{M_{1}, N_{1}}(\omega, t)-A_{M, N}(\omega, t)\right| \leq\left|\sum_{k=-M_{1}}^{-M-1} \mathrm{e}^{i \omega k} \frac{\sin (\pi(t-k))}{\pi(t-k)}\right| \\
+\left|\sum_{k=N+1}^{N_{1}} \mathrm{e}^{i \omega k} \frac{\sin (\pi(t-k))}{\pi(t-k)}\right|
\end{array}
$$

It is sufficient to analyze the case $t \notin \mathbb{Z}$, because $A_{M_{1}, N_{1}}(\omega, t)-$ $A_{M, N}(\omega, t)=0$ for $t \in \mathbb{Z},|t| \leq T$. The second term on the right hand side of (35) can be upper bounded by

$$
\left|\sum_{k=N+1}^{N_{1}} \mathrm{e}^{i \omega k} \frac{\sin (\pi(t-k))}{\pi(t-k)}\right| \leq\left|\sum_{k=N+1}^{N_{1}} \frac{\mathrm{e}^{i(\omega+\pi) k}}{\pi(t-k)}\right| .
$$

Defining $D_{k, N+1}(\omega)=\sum_{l=N+1}^{k} \mathrm{e}^{i(\omega+\pi) l}$ and using summation by parts in the same way as in (26), we have

$$
\sum_{k=N+1}^{N_{1}} \frac{\mathrm{e}^{i(\omega+\pi) k}}{k-t}=\frac{D_{N+1, N+1}}{N_{1}-t}+\sum_{k=N+1}^{N_{1}-1} \frac{D_{k, N+1}(\omega)}{(k-t)(k+1-t)}
$$

and since $\left|D_{k, N+1}(\omega)\right| \leq 1 /\left|\sin \left(\frac{\omega+\pi}{2}\right)\right|$, we obtain

$$
\begin{aligned}
& \left|\sum_{k=N+1}^{N_{1}} \frac{\mathrm{e}^{i(\omega+\pi) k}}{k-t}\right| \\
& \quad \leq \frac{1}{\left|\sin \left(\frac{\omega+\pi}{2}\right)\right|}\left(\frac{1}{N_{1}-t}+\sum_{k=N+1}^{N_{1}-1} \frac{1}{(k-t)(k+1-t)}\right) \\
& \quad \leq \frac{1}{\sin \left(\frac{\delta}{2}\right)}\left(\frac{1}{N-T}+\sum_{k=N+1}^{\infty} \frac{1}{(k-T)^{2}}\right) .
\end{aligned}
$$

The right-hand side of (37) converges to zero for $N \rightarrow \infty$. Thus, combining (36) and (37), we see that for all $\epsilon>0$ there exists a natural number $N_{0}=N_{0}(\epsilon, \delta, T)$ such that

$$
\left|\sum_{k=N+1}^{N_{1}} \mathrm{e}^{i \omega k} \frac{\sin (\pi(t-k))}{\pi(t-k)}\right|<\frac{\epsilon}{2}
$$

for all $N \geq N_{0}, N_{1}>N,|\omega| \leq \pi-\delta$, and $|t| \leq T$. The first term on the right-hand side of (35) can be treated in the same way.

Consequently, for all $\epsilon>0, T>0$, and $0<\delta<\pi$ there exist two natural numbers $N_{0}=N_{0}(\epsilon, \delta, T)$ and $M_{0}=M_{0}(\epsilon, \delta, T)$ such that for all $|\omega| \leq \pi-\delta$ and $|t| \leq T$, we have

$$
\left|A_{M_{1}, N_{1}}(\omega, t)-A_{M, N}(\omega, t)\right|<\epsilon
$$

for all $N \geq N_{0}, N_{1}>N$, and $M \geq M_{0}, M_{1}>M$. It follows that

$$
\lim _{N, M \rightarrow \infty} \max _{|t| \leq T} \max _{|\omega| \leq \pi-\delta}\left|\mathrm{e}^{i \omega t}-\sum_{k=-M}^{N} \mathrm{e}^{i \omega k} \frac{\sin (\pi(t-k))}{\pi(t-k)}\right|=0
$$

and, therefore, the assertion of the lemma is proved.

\section{REFERENCES}

[1] J. R. Higgins, "Five short stories about the cardinal series," Bull. Amer. Math. Soc., vol. 12, no. 1, pp. 45-89, 1985.

[2] C. E. Shannon, "Communication in the presence of noise," Proc. IEEE, vol. 72, no. 9, pp. 1152-1201, Sep. 1984, reprinted from the Proc. IRE, vol. 37, no. 1, pp. 1192-1201, Jan. 1949.

[3] A. J. Jerri, "The Shannon sampling theorem-Its various extensions and applications: A tutorial review," Proc. IEEE, vol. 65, no. 11, pp. 1565-1596, Nov. 1977.

[4] W. Chen, S. Itoh, and J. Shiki, "Irregular sampling theorems for wavelet subspaces," IEEE Trans. Inform. Theory, vol. 44, no. 3, pp. 1131-1142, May 1998.

[5] P. Zhao, C. Zhao, and P. G. Casazza, "Perturbation of regular sampling in shift-invariant spaces for frames," IEEE Trans. Inform. Theory, vol. 52, no. 10, pp. 4643-4648, Oct. 2006.

[6] Sampling Theory in Fourier and Signal Analysis-Advanced Topics, J. R. Higgins and R. L. Stens, Eds. Oxford, U.K.: Oxford Univ. Press, 1999.

[7] M. Zakai, "Band-limited functions and the sampling theorem," Inform. and Control, vol. 8, no. 2, pp. 143-158, 1965.

[8] A. A. V. Balakrishnan, "A note on the sampling principle for continuous signals," IRE Trans. Inform. Theory, vol. 3, no. 2, pp. 143-146, Jun. 1957.

[9] Y. K. Belyaev, "Analytic random processes," Theory of Prob. and Its Applic. vol. 4, no. 4, pp. 402-409, 1959.

[10] Z. A. Piranashvili, "On the problem of interpolation of random processes," Theory of Prob. and its Applic., vol. 4, no. 12, pp. 647-657, 1967.

[11] A. J. Lee, "Sampling theorems for nonstationary random processes," Trans. Amer. Mathemat. Soc., vol. 242, pp. 225-241, Aug. 1978.

[12] J. L. Brown, Jr., "Truncation error for band-limited random processes," Inform. Sci., vol. 1, pp. 261-171, 1969.

[13] S. Cambanis and E. Masry, "Truncation error bounds for the cardinal sampling expansion of band-limited signals," IEEE Trans. Inform. Theory, vol. IT-28, no. 4, pp. 605-612, Jul. 1982.

[14] C. Houdré, "Reconstruction of band limited processes from irregular samples," Annal. Probabil., vol. 23, no. 2, pp. 674-696, Apr. 1995.

[15] M. K. Habib, "Digital representations of operators on band-limited random signals," IEEE Trans. Inform. Theory, vol. 47, no. 1, pp. 173-177, Jan. 2001.

[16] A. J. Lee, "On band limited stochastic processes," SIAM J. Appl. Math., vol. 30 , no. 2 , pp. 269-277, Mar. 1976. 
[17] H. Boche and U. J. Mönich, "General behavior of sampling-based signal and system representation," in Proc. 2008 IEEE Int. Symp. Information Theory, Jul. 2008, pp. 2439-2443.

[18] P. L. Butzer, W. Splettstößer, and R. L. Stens, "The sampling theorem and linear prediction in signal analysis," Jahresbericht der Deutschen Mathematiker-Vereinigung, vol. 90, no. 1, pp. 1-70, Jan. 1988.

[19] C. J. Standish, "Two remarks on the reconstruction of sampled nonbandlimited functions," IBM J. Res. Develop., vol. 11, no. 6, pp. 648-649, Nov. 1967.

[20] J. L. Brown, Jr., "Bounds for truncation error in sampling expansions of band-limited signals," IEEE Trans. Inform. Theory, vol. 15, no. 4, pp. 440-444, Jul. 1969.

[21] M. Loève, Probability Theory, 3rd ed. New York: Van Nostrand, 1963.

[22] H. Cramér and M. R. Leadbetter, Stationary and Related Stochastic Processes. Mineola, NY: Dover Publications, 2004.

[23] H. Boche and U. J. Mönich, "On the behavior of Shannon's sampling series for bounded signals with applications," Signal Process., vol. 88, no. 3, pp. 492-501, Mar. 2008.

[24] J. S. Howland, Basic Real Analysis. Bloomfield, NJ: Jones \& Bartlett, 2009.

[25] H. D. Helms and J. B. Thomas, "Truncation error of sampling-theorem expansions," Proc. IRE, vol. 50, no. 2, pp. 179-184, Feb. 1962.

[26] K. Yao and J. B. Thomas, "On truncation error bounds for sampling representations of band-limited signals," IEEE Trans. Aerosp. Electron. Syst., vol. AES-2, no. 6, pp. 640-647, Nov. 1966.

[27] W. Rudin, Real and Complex Analysis, 3rd ed. New York: McGrawHill, 1987

[28] I. S. Gradshteyn and I. M. Ryzhjk, Table of Integrals, Series, and Products, A. Jeffrey, Ed., 6th ed. New York: Academic, 2000.

[29] H. Boche and U. J. Mönich, "Non-uniform sampling-Signal and system representation," in Proc. 2008 Int. Symp. Information Theory and Its Applications (ISITA2008), Auckland, New Zealand, Dec. 2008, pp. 1576-1581.
Holger Boche (M'04-SM'07) received his Dipl.-Ing. and Dr.-Ing. degrees in electrical engineering from the Technische Universität Dresden, Germany, in 1990 and 1994, respectively. In 1992 he graduated in mathematics from the Technische Universität Dresden, and in 1998 he received his Dr.rer.nat. degree in pure mathematics from the Technische Universität Berlin. From 1994 to 1997, he did postgraduate studies in mathematics at the Friedrich-Schiller Universität Jena, Germany. In 1997, he joined the Heinrich-Hertz-Institut (HHI) für Nachrichtentechnik Berlin. Since 2002, he has been Full Professor for Mobile Communication Networks at the Technische Universität Berlin at the Institute for Communications Systems. In 2003, he became Director of the Fraunhofer German-Sino Lab for Mobile Communications, Berlin, Germany, and since 2004 he has also been Director of the Fraunhofer Institute for Telecommunications (HHI), Berlin, Germany. He was Visiting Professor at the ETH Zurich during winter term 2004 and 2006 and at KTH Stockholm during summer term 2005. Prof. Boche received the Research Award "Technische Kommunikation" from the Alcatel SEL Foundation in October 2003, the "Innovation Award" from the Vodafone Foundation in June 2006, and the Gottfried Wilhelm Leibniz Prize from the Deutsche Forschungsgemeinschaft (German Research Foundation) in 2008. He was co-recipient of the 2006 IEEE Signal Processing Society Best Paper Award and recipient of the 2007 IEEE Signal Processing Society Best Paper Award. He is a member of IEEE Signal Processing Society SPCOM and SPTM Technical Committee. He was elected a member of the German Academy of Sciences (Leopoldina) in 2008 and of Berlin Brandenburg Academy of Sciences and Humanities in 2009.

Ullrich J. Mönich (S'06) received the Dipl--Ing. degree in electrical engineering from the Technische Universität Berlin, Germany in 2005, where he is currently pursuing the Ph.D. degree. During winter term 2003, he was a visiting researcher at the University of California, Santa Barbara. His research activities comprise sampling theory, signal processing, and information theory. 